\title{
Irritable Bowel Syndrome and Nickel Allergy: What Is the Role of the Low Nickel Diet?
}

\begin{abstract}
Angela Rizzi, ${ }^{1 *}$ Eleonora Nucera, ${ }^{1}$ Lucrezia Laterza, ${ }^{2}$ Eleonora Gaetani, ${ }^{2}$ Venanzio Valenza, ${ }^{3}$ Giuseppe M Corbo, ${ }^{4}$ Riccardo Inchingolo, ${ }^{4}$ Alessandro Buonomo, ${ }^{1}$ Domenico Schiavino, ${ }^{1}$ and Antonio Gasbarrini ${ }^{2}$

${ }^{1}$ Allergy Unit, Department of Rheumatology, Immunology, Dermatology and Uro-Nefrological Sciences, Fondazione Policlinico Universitario A. Gemelli, Università Cattolica del Sacro Cuore, Rome, Italy; ${ }^{2}$ Division of Gastroenterology, Department of Internal Medicine, Fondazione Policlinico Universitario A. Gemelli, Università Cattolica del Sacro Cuore, Rome, Italy; ${ }^{3}$ Department of Nuclear Medicine, Fondazione Policlinico Universitario A. Gemelli, Università Cattolica del Sacro Cuore, Rome, Italy; and ${ }^{4}$ Department of Pulmonary Medicine, Fondazione Policlinico Universitario A. Gemelli, Università Cattolica del Sacro Cuore, Rome, Italy
\end{abstract}

\section{Background/Aims}

Irritable bowel syndrome (IBS) is characterized by chronic abdominal pain or discomfort accompanied by abnormal bowel movements. In sensitized subjects, ingested nickel (Ni) may induce gastrointestinal symptoms similar to IBS, in addition to typical systemic cutaneous lesions (systemic nickel allergy syndrome [SNAS]). A low nickel diet could improve the systemic manifestations. We evaluated prevalence of nickel allergy in IBS and effects of low Ni diet on (1) gastrointestinal symptoms control, (2) intestinal barrier function, (3) quality of life, and (4) psychological status of patients with IBS and Ni-sensitized patients.

\section{Methods}

Twenty consecutive patients affected by IBS and suspected SNAS underwent intestinal permeability tests. Gastrointestinal symptoms were evaluated using the visual analogue scale before and after 3 months low Ni diet. Subjects with increased intestinal permeability at baseline repeated nuclear examination after the diet.

\section{Results}

The most frequent profile was diarrhea-predominant IBS (8/20). The low Ni diet induced a significant and constant improvement of gastrointestinal symptoms and an equally significant improvement of visual analogue scale. Mean urinary output of ${ }^{51} \mathrm{Chromium}$ ethylene-diamine-tetra-acetate $\left({ }^{51} \mathrm{Cr}\right.$-EDTA) was $5.91 \% / 24 \mathrm{hr}( \pm 2.08)$, significantly different from the control group $(2.20 \% / 24 \mathrm{hr} \pm$ $0.60, P<0.0001)$.

\section{Conclusion}

This pilot study shows that low Ni diet improves gastrointestinal symptoms in patients with IBS and SNAS.

(J Neurogastroenterol Motil 2017;23:101-108)

\section{Key Words}

Diet; Hypersensitivity; Irritable bowel syndrome; Nickel; Permeability

Received: February 22, 2016 Revised: July 4, 2016 Accepted: October 23, 2016

(ङ) This is an Open Access article distributed under the terms of the Creative Commons Attribution Non-Commercial License (http://creativecommons. org/licenses/by-nc/4.0) which permits unrestricted non-commercial use, distribution, and reproduction in any medium, provided the original work is properly cited.

${ }^{*}$ Correspondence: Angela Rizzi, MD, PhD

Allergy Unit, Department of Rheumatology, Immunology, Dermatology and Uro-Nefrological Sciences, Fondazione Policlinico Universitario A. Gemelli, Università Cattolica del Sacro Cuore, Largo A. Gemelli 8, 00168 Rome, Italy

Tel: +39-06-30155896, Fax: +39-06-30156999, E-mail: angelarizzi81@gmail.com

Angela Rizzi and Eleonora Nucera contributed equally to this work.

This work was accepted as an abstract at the United European Gastroenterology Week in Vienna 2014 and at the 20th National Congress of Digestive Diseases, Italian Federation of Societies of Digestive Diseases - FISMAD in Naples 2014. 
psychological state and the quality of life in this cohort.

\section{Introduction}

Irritable bowel syndrome (IBS) is a functional gastrointestinal disorder, characterized by the presence of chronic abdominal pain or abdominal discomfort associated with altered bowel function and bloating. ${ }^{1}$ It is probably the most frequent cause of referral to gastroenterologists and also the most common disorder encountered by psychiatrists seen in primary care. However, many patients do not seek medical care for symptoms indicative of IBS and consequently the true incidence of IBS may be underestimated. ${ }^{2}$ Several studies have demonstrated that patients with IBS have a significantly impaired health-related quality of life and reduced work productivity. ${ }^{3}$

The mechanisms behind the disturbances are thought to be a result of dysregulation of the "brain-gut axis" (in terms of enhanced motility, abnormal sensation, and autonomic reactivity), a chronic low-grade inflammation within the gut wall, and altered immunological function. ${ }^{4}$ To date, there are no structural or biochemical disease markers for these patients. Therefore, clinical diagnostic criteria have been used to define IBS in the absence of organic disorders (Rome criteria). ${ }^{1}$

In sensitized subjects, the ingested nickel (Ni) may induce gastrointestinal symptoms like those encountered in IBS (nausea, pyrosis, meteorism, abdominal pain, diarrhea, and constipation), in addition to typical systemic cutaneous lesions. This clinical picture is known as systemic nickel allergy syndrome (SNAS). ${ }^{5}$ Although in the literature there is no general agreement about the efficacy of a dietary treatment, ${ }^{6}$ some studies have confirmed improvement of dermatitis on $\mathrm{Ni}$ free or a low $\mathrm{Ni}$ diet for a minimum period of 4 weeks (up to a maximum of 6 months).

Growing evidence suggests that patients with IBS have decreased intestinal barrier function and that some forms of IBS are associated with low-grade intestinal inflammation. ${ }^{8}$ Similarly, Ni allergy is associated with immune system dysregulation with prevalent immunosuppressive action. In particular, Ni-sensitive patients with symptom recrudescence after $\mathrm{Ni}$ ingestion showed significant decrease of $\mathrm{CD} 8+$ and massive inflammatory infiltration of $\mathrm{CD} 4+$ $\mathrm{CD} 45 \mathrm{RO}+\mathrm{T}$ lymphocytes in the duodenal lamina propria and epithelium $^{9}$ as well as an involvement of Th2-type cytokines (IL-5 and IL-13). ${ }^{10}$

The aim of this prospective, single-center, pilot study was to assess: (1) the prevalence of Ni allergy in IBS patients, (2) the clinical response to low $\mathrm{Ni}$ diet in IBS patients with concomitant $\mathrm{Ni}$ sensitization, (3) the intestinal permeability (IP) before and after low Ni diet compared with matched healthy controls, and finally, (4) the

\section{Materials and Methods}

\section{Participants}

Between December 2012 and January 2014, subjects with IBS, not enrolled in other studies and referred to the Gastroenterology Division of Fondazione Policlinico Universitario A. Gemelli, Università Cattolica del Sacro Cuore, Rome, Italy, were evaluated for eligibility. They were selected on the presence of abdominal pain and/or discomfort with disturbed defecation (Rome III criteria), ${ }^{11}$ in absence of obvious alarm features. Subsequently, patients with IBS were classified as diarrhea-predominant (IBS-D), constipationpredominant (IBS-C), mixed (both diarrhea and constipation, IBS$\mathrm{M})$, and unsubtyped (neither diarrhea nor constipation, IBS-U). ${ }^{1}$

Exclusion criteria included: age $<18$ years and $>75$ years; other organic gastrointestinal diseases, such as peptic ulcer, inflammatory bowel diseases, celiac disease, gastrointestinal infections, and small intestinal bacterial overgrowth; diabetes mellitus, hepatic, renal or cardiac dysfunction, and thyroid disease or tumor; intake of non steroidal anti-inflammatory agents and other medications known to affect IP in the previous 4 weeks; and pregnancy, lactation, smoking, abuse of alcohol, coffee, tea, and cola intake.

In order to exclude organic diseases, all screened IBS patients underwent stool cultures, parasitological stool exam, fecal calprotectine, cell blood count, erythrocyte sedimentation rate, C-reactive protein, and serological tests for celiac disease. Patients with high fecal calprotectine underwent colonoscopy to exclude inflammatory bowel disease. Patients with a diagnosis of lactose intolerance, confirmed by a breath test, who followed a lactose-free diet for at least 4 weeks but still experienced symptoms, could be enrolled if they are willing to be adherent to a lactose-free diet.

\section{Study Protocol}

All patients who satisfied the aforementioned selection criteria were examined in the Allergy Unit of our hospital, where they underwent an allergological work up including patch tests with $5 \% \mathrm{Ni}$ sulfate in petrolatum and prick tests in order to exclude food allergies.

IBS patients with concomitant positive Ni patch tests were asked to respond to the Italian version of following questionnaires: visual analogue scale (VAS) for IBS (VAS-IBS), VAS, short form 36 items health survey (SF-36), Psychological General Well Being Index (PGWBI), and Minnesota Multiphasic Personality Inven- 
tory (MMPI-2) ${ }^{51}$ Chromium ethylene-diamine-tetra-acetate $\left({ }^{51} \mathrm{Cr}\right.$ EDTA) absorption test was performed in all patients to assess IP in the Nuclear Medicine Unit of our hospital. Patients were matched with the control population consisting of healthy subjects without a history of IBS, who were similar with patients for sex, age, and social background. Afterward, the patients were recommended to follow a diet with a low $\mathrm{Ni}$ content for 3 months. The patients wrote a dietary diary, and compliance to the elimination diet was evaluated by trained dieticians.

At the end of 3 months, all patients repeated the questionnaires that measured physical, mental and social functioning, while only those with altered permeability at baseline repeated nuclear study. Controls were not used for the rest of the analysis other than the IP study.

All subjects gave written informed consent prior to starting the study. The study received approval by the Institutional Review Board since it conformed to the ethical guidelines of the 1975 Declaration of Helsinki (6th revision, 2008).

All patients underwent the skin-patch test with $5 \% \mathrm{Ni}$ sulfate $\left(\mathrm{NiSO}_{4}\right)$ in petrolatum (Hermal, Hamburg, Germany) according to International Contact Dermatitis Research Group Guidelines. ${ }^{12}$ Patch tests were evaluated 48 hours and 72 hours after their application and were considered positive if an eczematous-vesicular reaction occurred at the contact site with the allergen. The intensity was assessed with the following criteria $^{13}$ : (1) \pm , faint and non-palpable erythema; (2) + , palpable erythema; $(3)++$, strong infiltrate, numerous papules, vesicles present, and strong reaction; and (4) +++ , coalescing vesicles, bullae or ulceration extreme reaction erythema.

\section{Interventional Diet}

The absolute removal of $\mathrm{Ni}$ from the diet is impractical because of its ubiquitous presence in almost all foods; therefore, we excluded all foods with a high content of $\mathrm{Ni}(\mathrm{Ni} 100 \mu \mathrm{g} / \mathrm{kg}-\mathrm{Ni}>500 \mu \mathrm{g} /$ $\mathrm{kg}$ ) following BraMa-Ni $\operatorname{diet}^{14}$ as a guide for 12 weeks (Table 1). Furthermore, patients were asked to avoid the use of stainless-steel utensils to reduce Ni contamination during cooking.

\section{Questionnaires}

We used VAS-IBS to assess the presence and severity of symptoms related to IBS and the improvements obtained by the dietary treatment. Abdominal bloating, pain/discomfort, flatulence, abdominal cramps, constipation, diarrhea, nausea, and vomiting were evaluated. In particular, patients were asked to assign each symptom a score from 0 to $10(0=$ absence of symptom; $10=$ highest expression of the symptom). ${ }^{15}$

VAS was evaluated as visual representation of the possible total clinical improvement achieved by diet therapy. The amplitude is represented by a line, usually $10 \mathrm{~cm}$ long without notches at each centimeter. One end indicates no benefit, while the other is an excellent benefit. Moreover, we adopted the SF-36 as generic measure of perceived health status that incorporates behavioral functioning,

Table 1. Nickel-rich Foods

\begin{tabular}{llll}
\hline Ni $100 \mu \mathrm{g} / \mathrm{kg}$ & Ni $200 \mu \mathrm{g} / \mathrm{kg}$ & Ni $500 \mu \mathrm{g} / \mathrm{kg}$ & $\mathrm{Ni}>500 \mu \mathrm{g} / \mathrm{kg}$ \\
\hline Carrots & Apricots & Artichoke & Almonds \\
Figs & Broccoli & Asparagus & Cocoa \\
Lettuce & Corn & Beans & Concentrated tomato \\
Green salad & Lobster & Cabbage & Lentils \\
Licorice & Onions & Cauliflower & Oats \\
Mushrooms & Pears & Green beans & Peanuts \\
Plaice and cod & Raisins & Integral flour & Walnuts \\
Rhubarb & & Yeast & Margarine \\
Tea & & Mussels & Oysters \\
& & Potatoes & Peas \\
& & Plums & \\
& & Spinach & \\
\end{tabular}

$\mathrm{Ni}$, nickel. 
subjective well-being and perception of health by assessing 8 health concepts: physical function (how patients perceive their ability to perform physical tasks), role-physical (how patients perceive their ability to fulfill their life role physically), bodily pain (how patients perceive their level of pain), general health (how patients perceive their overall health and well-being), vitality (how patients perceive their level of "energy"), social function (how patients perceive their ability to participate in social activities), role-emotional (how patients perceive their ability to fulfill their life role emotionally), and mental health (how patients perceive their emotional and psychological well-being). The scores on all scales ranged from 0 to 100 , with higher scores reflecting better health. ${ }^{16}$

All patients answered the PGWBI questionnaire consisting of 22 items, each of which presents a series of 6 possible responses whose score is proportional to the positivity of "well being" reported during the last 4 weeks and variable between "0" (the worst condition) and " 110 " (the best condition). The scale also consists of 6 domains or dimensions: anxiety, depression, positive and well-being, self-control, general health, and vitality. ${ }^{17}$

Finally, we used the MMPI-2 questionnaire containing 567 items with 2 choices of answer ("true" or "false") ${ }^{18}$ in order to assess the main structural features of personality and emotional disorders.

\section{Assessment of Intestinal Permeability}

IP was assessed by the ${ }^{51} \mathrm{Cr}$-EDTA permeability test. ${ }^{19}$

After an overnight fast, patients drank $2.96 \mathrm{MBq}{ }^{51} \mathrm{Cr}$-EDTA (specific activity 1-2 $\mathrm{mCi} / \mathrm{mg}$ chromium; Amersham Health, England, UK) in $10 \mathrm{~mL}$ water, and urine was collected for the next 24 hours. Two 3-mL samples of the collected urine were analyzed using a $\gamma$-counter (LKB-Wallac 1282 Compugamma, Turku, Finland), as described previously. ${ }^{20}$

${ }^{51} \mathrm{Cr}$-EDTA clearance was calculated using the following formula:

$[$ (mean urinary counts $\times$ urinary volume $) \times$

(standard counts $\times 50)]-1$

The results were expressed as percentages of the ingested dose and considered indicative of altered IP when ${ }^{51} \mathrm{Cr}$-EDTA was $\geq 3 \%{ }^{20}$

\section{Statistical Methods}

The socio-demographic and clinical characteristics of the studied population were reported as means and standard deviations for continuous variables and as frequencies and percentages for cate- gorical variables. The Student two-tailed $t$ test was used to compare the values of IP, VAS, VAS-IBS, SF-36, and PGWBI before and after diet therapy.

Regression analysis was performed in order to investigate the best prediction of the dependent variable (the difference between post- and pre-diet ${ }^{51} \mathrm{Cr}$-EDTA excretion) based on the data set including the severity of allergic skin reaction to $\mathrm{Ni}$ and the IBS subtype as independent variables, adjusting for atopy, age, and gender. The $\beta$ coefficient of the linear regression analyses specifies how much the dependent variable changes for a given change in each independent variable. A $P$-value $<0.05$ was considered statistically significant. Statistical analysis was performed using SPSS version 16.0 .

\section{Results}

Fifty patients fulfilling the Rome III diagnostic criteria for IBS were screened. During clinical work-up, 21 were excluded: 8 for non-sensitization to $\mathrm{Ni}$ (negative $\mathrm{Ni}$ patch tests), 7 for concomitant organic disease (2 celiac disease, 1 polycystic ovary syndrome, 2 small bowel bacterial overgrowth, 1 stomach cancer, and 1 intestinal candidiasis), 6 for denied consent to the repetition of IP test. Finally, 9 patients dropped out without giving reason.

The demographic, clinical, and social aspects of the protocol population are described in Table 2 . The mean age was $42 \pm 14$ years, and there was a predominance of women. The majority of enrolled patients was affected by the IBS-D profile. There was also a significant presence of lactose malabsorption (15/20). Half of the patients showed allergic contact dermatitis towards other haptens (patch test positive for palladium, cobalt, Kathon). The detection of atopy was sporadic. Education was measured by the number of years of completed school education (years at school): $65 \%$ of patients had a higher level of education (average schooling of 13 years). About two-thirds of the sample was performing work outside the home. Regarding marital status, $50 \%$ of the subjects was married. There was high percentage of skin symptoms (itching and rash) and headache (Table 2).

The low Ni diet determined a remarkable improvement of gastrointestinal symptoms, except for vomiting (Fig. 1), and an equally significant improvement of VAS, expression of clinical efficacy achieved (average pre-diet: 3.9 , average post-diet:7.3; $P<0.0001$ ).

Finally, as regards to the psychometric indices study changes after the low Ni diet, there was a trend of increase of all indices with statistically significant change for the bodily pain scale (average prediet: 45.5 , average post-diet: $50.9 ; P=0.028$ ) and physical compo- 
Table 2. Demographic Characteristics of Study Population, Reported at the First Visit

\begin{tabular}{lc}
\hline \multicolumn{1}{c}{ Characteristics } & $\mathrm{n}=20$ \\
\hline Sex $(\mathrm{M} / \mathrm{F}, \mathrm{n})$ & $3 / 17$ \\
Age (mean $\pm \mathrm{SD}, \mathrm{yr})$ & $42 \pm 14$ \\
BMI (mean $\left.\pm \mathrm{SD}, \mathrm{kg} / \mathrm{m}^{2}\right)$ & $23 \pm 4$ \\
Irritable bowel syndrome (n [\%]) & \\
IBS-D & $8(40)$ \\
IBS-C & $4(20)$ \\
IBS-M & $6(30)$ \\
IBS-U & $2(10)$ \\
Patch Test (n [\%]) & \\
Grade A $( \pm)$ & 6 \\
Grade B (+) & $6(30)$ \\
Grade C (++) & $9(45)$ \\
Grade D (+++) & $5(25)$ \\
Atopy (n [\%]) & $4(20)$ \\
Lactose Intolerance (n [\%]) & $15(75)$ \\
Married (n [\%]) & $10(50)$ \\
High education (n [\%] $)^{\mathrm{a}}$ & $13(65)$ \\
Others symptoms (n [\%]) & \\
Localized itching & $15(75)$ \\
Generalized itching & $9(45)$ \\
Cutanoeus rash & $15(75)$ \\
Headache & $11(55)$ \\
Cough & $7(35)$ \\
\hline
\end{tabular}

${ }^{\text {a} H i g h ~ e d u c a t i o n ~ m e a n s ~ h i g h ~ s c h o o l ~ d i p l o m a ~ a n d ~ u n d e r g r a d u a t e / g r a d u a t e ~}$ studies.

M, male; F, female; BMI, body mass index; IBS, irritable bowel syndrome; IBS-D, diarrhea-predominant IBS; IBS-C, constipation-predominant IBS; IBS-M, mixed (both diarrhea and constipation) IBS; IBS-U, unsubtyped (neither diarrhea nor constipation) IBS

nent summary (average pre-diet: 46.8, average post-diet: $51.9 ; P=$ 0.018) (Fig. 2).

The study of the main structural features of personality and emotional disorders revealed a high prevalence of psychiatric symptoms in our population. The analysis of the distribution of code types with Welsh's system led us to observe a wide heterogeneity of clinical personality traits with prevalence of anxiety profile (Fig. 3).

The study of IP with ${ }^{51} \mathrm{Cr}$-DTPA showed a mean value of urinary excretion of $5.91 \% / 24 \mathrm{hr}( \pm 2.08)$, which was significantly different from the control group $(2.20 \% / 24 \mathrm{hr} \pm 0.60, P<0.0001)$. All patients had an altered IP. No IP values returned to normal after diet therapy, regardless of IBS profile and severity of $\mathrm{Ni}$ allergy.

Subsequently, the distribution of permeability values changes was evaluated. We analysed "delta permeability" ( $\left.\Delta^{51} \mathrm{Cr}-\mathrm{DTPA}\right)$ defined as the difference between post- and pre-diet urinary excretion of chromium. We observed a dual and opposite trend of $\Delta^{51} \mathrm{Cr}$ -

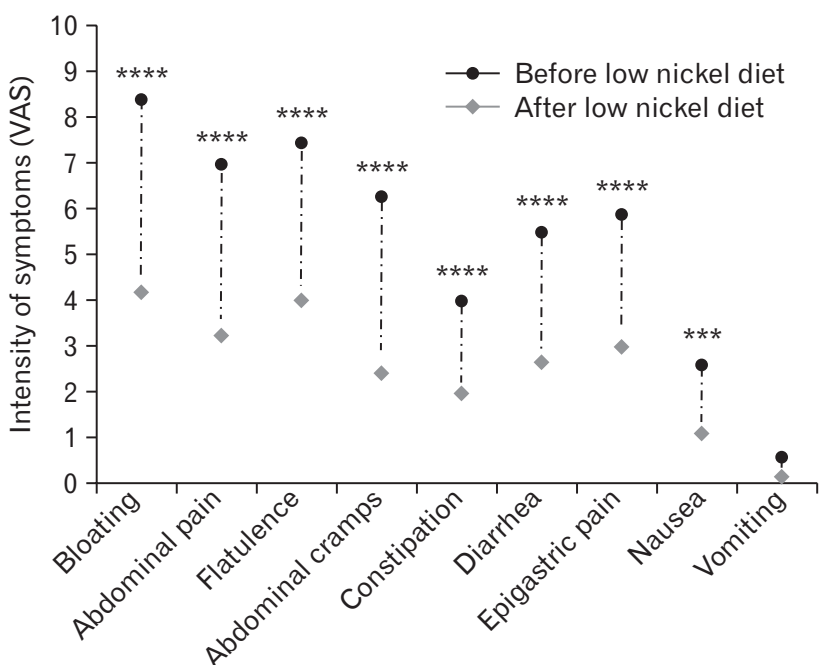

Figure 1. Changes in visual analogue scale (VAS) for irritable bowel syndrome, comparing before and after 3 months of low nickel diet. The circles indicate the mean values of symptoms considered before treatment; the grey diamonds indicate mean values of the same symptoms after treatment. ${ }^{* * *} P<0.001,{ }^{* * * *} P<0.0001$.

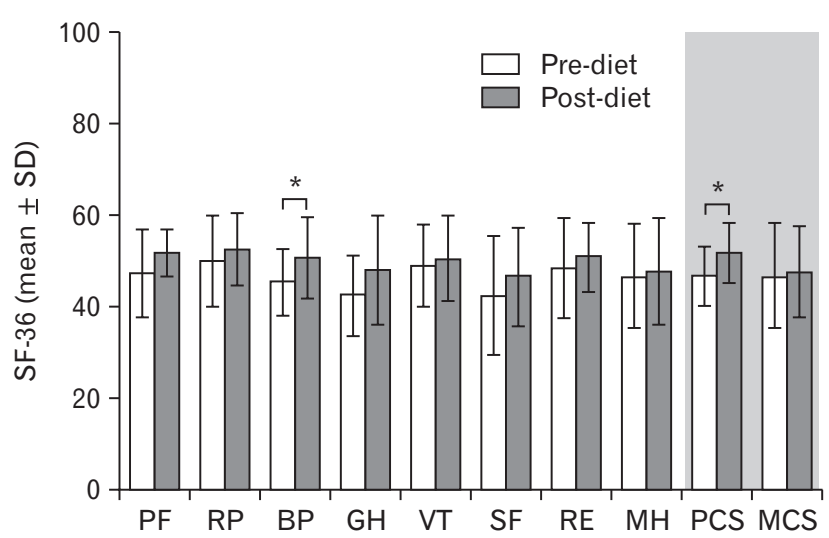

Figure 2. Changes of short form 36 items health survey (SF-36) psychometric indices after low nickel diet. PF, physical function; RP, role-physical; BP, bodily pain; GH, general health; VT, vitality; SF, social function; RE, role-emotional; $\mathrm{MH}$, mental health; PCS, physical component summary; MCS, mental component summary. ${ }^{*} \mathrm{P}<$ 0.05 .

DTPA: half of the enrolled patients showed a statistically significant reduction in urinary excretion of the tracer $(P=0.028)$, whereas the remaining 10 patients showed a worsening of the gut-barrier function $(P=0.0001)$.

The study of the determinants of IP change ( $\left.\Delta^{51} \mathrm{Cr}-\mathrm{DTPA}\right)$, through linear regression analysis, showed a direct relation with moderate $(++)(P=0.057)$ and strong $(+++)(P=0.026)$ al- 


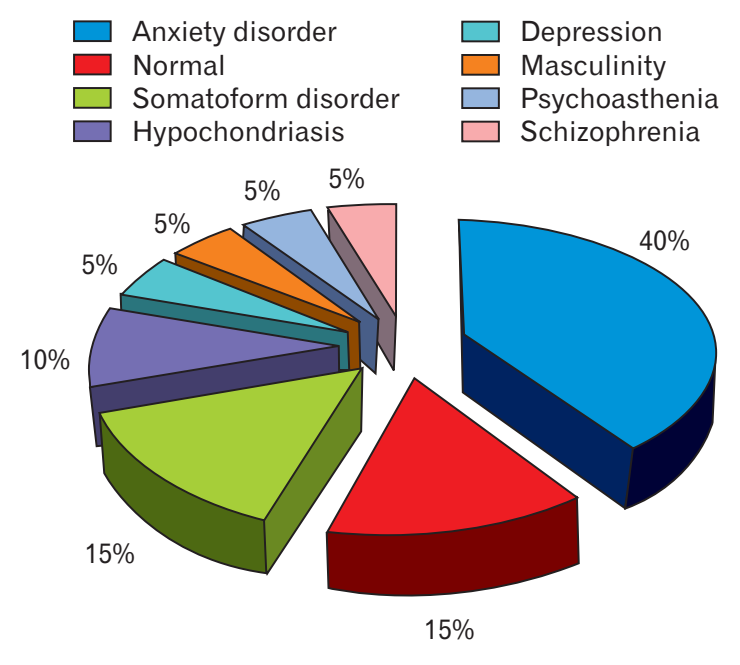

Figure 3. The analysis of the distribution of code types with Welsh's system.

lergic reactions to $\mathrm{Ni}$. Whereas, an indirect relationship was found with IBS-M $(P=0.010)$ and IBS-U subtypes $(P=0.017)$, adjusting for age, gender, and the presence of atopy (Table 3 ).

\section{Discussion}

Our data demonstrate that: (1) Ni allergy is highly prevalent in patients affected by IBS, with a higher frequency in females; (2) a low $\mathrm{Ni}$ diet determines a clear improvement on both gastrointestinal symptoms and state of general physical health in IBS patients with concomitant Ni allergy; and (3) the change of IP after low Ni diet is influenced by skin reactivity to $\mathrm{Ni}$ and IBS subtypes.

To our knowledge, this is the first report showing high prevalence of Ni allergy in patients with IBS. These findings have led investigators to develop hypotheses regarding underlying pathophysiological mechanisms connecting these disorders. In fact, it is known that the ingested $\mathrm{Ni}$ can be responsible for gastrointestinal symptoms mimicking the clinical features of IBS. ${ }^{21}$ This is especially true in those patients who underwent a long series of gastrointestinal tests without objective signs of organic pathology. Moreover, postprandial worsening of symptoms, as well as adverse reactions to one or more foods is common in IBS patients. ${ }^{22}$ Certainly, consistent with previous trials, we found female predominance, supporting the idea that gender aspects have to be taken into account when food related GI symptoms are evaluated. ${ }^{23}$

Historically, diet has been assigned a relatively minor role in the pathogenesis of IBS. ${ }^{24}$ Nonetheless, the proposals of dietary intervention in the management of IBS are continuously on the rise.
Table 3. Determinants of Change of Intestinal Permeability $\left(\triangle^{51} \mathrm{Cr}\right.$ DTPA) Calculated by Linear Regression Analysis

\begin{tabular}{lcc}
\hline & \multicolumn{2}{c}{$\Delta^{51}$ Cr-DTPA } \\
\cline { 2 - 3 } & Coeff. & $P$-value \\
\hline Grade B patch test $(+)$ & Ref $^{\mathrm{a}}$ & - \\
Grade C patch test $(++)$ & 2.375 & 0.057 \\
Grade D patch test $(+++)$ & 3.350 & 0.026 \\
IBS-D & Ref $^{\text {a }}$ & - \\
IBS-C & -0.209 & 0.854 \\
IBS-M & -3.183 & 0.010 \\
IBS-U & -3.924 & 0.017 \\
Atopy & 1.518 & 0.165 \\
Age & 0.057 & 0.128 \\
Sex & -0.210 & 0.867 \\
\hline
\end{tabular}

${ }^{a}$ Reference group of linear regression.

IBS, irritable bowel syndrome; IBS-D, diarrhea-predominant IBS; IBS-C, constipation-predominant IBS; IBS-M, mixed (both diarrhea and constipation) IBS; IBS-U, unsubtyped (neither diarrhea nor constipation) IBS.

$\triangle^{51} \mathrm{Cr}$-DTPA defined as the difference between post- and pre-diet urinary excretion of chromium.

In the early 2000s, the fermentable oligo-, di- and mono saccharides and polyols (FODMAP) theory was introduced. It proposes that specific nondigested or poorly digested carbohydrates contribute to common IBS symptoms and induce alterations in gut motility and secretion. ${ }^{25}$

Low-FODMAP diets have been shown to reduce gastrointestinal symptoms if compared with both high-FODMAP diets ${ }^{25}$ and National Institute for Health and Clinical Excellence standard dietary advice. $^{26}$ The efficacy of low-FODMAP diet has been questioned in a recent multi-center, parallel, single-blind RCT that showed no superiority of low-FODMAP diet compared to traditional dietary advice in improving IBS symptoms. ${ }^{27}$

Finally, the British Dietetic Association also recommends that dietary and lifestyle advice should be routinely provided to patients with IBS. ${ }^{28}$

On the other hand, the impact of food allergy in the clinical management of IBS is more controversial and less defined. ${ }^{24}$

Our study introduces a new dietary strategy suitable for a significant proportion of IBS patients: those with concomitant Ni sensitization. The low Ni diet improved significantly all gastrointestinal symptoms evaluated except vomiting, symptom not so common in IBS patients. The pathophysiological basis of this improvement is unknown. As hypothesized by other authors, ${ }^{10,29}$ we think that the almost complete nickel sulfate removal could explain the clinical benefits through a reduction of pro-inflammatory state induced by $\mathrm{Ni}$.

In order to evaluate the effects of low $\mathrm{Ni}$ diet in this subset of 
IBS patients, we used the study of IP. As expected, barrier gut function was compromised in all patients and it did not return to normal after diet therapy. This is due to multifactorial etiopathogenesis of impairment of IP in IBS. ${ }^{30}$ The study of the determinants of change of IP after low Ni diet highlights the different role of severity of Ni reactivity and IBS subtypes. In particular, the evidence of direct relationship of change of IP after diet with more severe allergic reactions to $\mathrm{Ni}$ suggests that the allergic status of the intestinal mucosa remains present even long after the last allergen exposure and facilitates the worsening of intestinal barrier dysfunction.

Conversely, some IBS subtypes (IBS-M and IBS-U) with concomitant $\mathrm{Ni}$ sensitization seem to benefit from low $\mathrm{Ni}$ diet in terms of reduction of IP after dietary management. If this apparently "protective" role of these subtypes is an epiphenomena or a step of pathophysiological amelioration of intestinal barrier function in IBS patients with concomitant Ni allergy remains unknown, we think that this could be worthy of future studies.

It is known that psychological distress is common in IBS patients compared to the general population. ${ }^{31,32}$ Our study confirms the high prevalence of psychiatric symptoms, particularly anxiety, among IBS patients with concomitant Ni allergy.

This study has few limitations. First, it was conducted at a tertiary care center in which we perform many clinical research studies. Therefore, the apparently limited number of enrolled patients is due to the highly selective criteria. The other major shortcoming concerns the design of the study. We had a control group only for studying permeability, while all other outcomes were compared among the intra-groups and the patients served as their own control. However, the use of daily diary cards helped to obviate subjective interpretation or recall bias by providing a systematic and objective measure of daily bowel habits.

Despite these limitations, this study found objective evidence that individuals initiating a Ni dietary avoidance had a profound clinical response in their IBS symptoms confirming the link between IBS and Ni sensitivity. Furthermore, low Ni diet allowed many foods usually avoided in a low-FODMAP diet.

This study shows a high prevalence of $\mathrm{Ni}$ allergy in patients with IBS and highlights the usefulness of clinical indicators, based on a broad symptom score, as the primary monitors to evaluate the response to dietary treatment. Furthermore, it confirms that the study of IP is a crucial step in the clinical management of IBS patients in particular with associated $\mathrm{Ni}$ allergy. However, due to the high response of IBS patients to dietary restrictions and the possible placebo/nocebo effect, these results should be confirmed by a large RCT.

\section{Financial support: None.}

\section{Conflicts of interest: None.}

Author contributions: Angela Rizzi: conception and design, execution study design, acquisition of data, and manuscript writing; Eleonora Nucera and Antonio Gasbarrini: conception and design, execution study design, and manuscript revision; Lucrezia Laterza, Alessandro Buonomo, and Venanzio Valenza: acquisition of data; Eleonora Gaetani: data interpretation; Giuseppe M Corbo: statistical analysis; Riccardo Inchingolo: data interpretation and manuscript writing; and Domenico Schiavino: manuscript revision.

\section{References}

1. Longstreth GF, Thompson WG, Chey WD, Houghton LA, Mearin F, Spiller RC. Functional bowel disorders. Gastroenterology 2006;130:1480-1491.

2. Oshima T, Miwa H. Epidemiology of functional gastrointestinal disorders in Japan and in the world. J Neurogastroenterol Motil 2015;21:320329.

3. Lackner JM, Gudleski GD, Thakur ER, Stewart TJ, Iacobucci GJ, Spiegel BM. The impact of physical complaints, social environment, and psychological functioning on IBS patients' health perceptions: looking beyond GI symptom severity. Am J Gastroenterol. 2014;109:224-233.

4. Ohman L, Simrén M. Pathogenesis of IBS: role of inflammation, immunity and neuroimmune interactions. Nat Rev Gastroenterol Hepatol 2010;7:163-173

5. Falagiani P, Di Gioacchino M, Ricciardi L, et al. Systemic nickel allergy syndrome (SNAS): a review. Rev Port Imunoalergologia 2008;16:135147

6. Pizzutelli S. Systemic nickel hypersensitivity and diet: myth or reality? Eur Ann Allergy Clin Immunol 2011;43:5-18

7. Veien NK, Menné T. Nickel contact allergy and a nickel-restricted diet. Semin Dermatol. 1990; 9:197-205.

8. Piche T, Barbara G, Aubert P, et al. Impaired intestinal barrier integrity in the colon of patients with irritable bowel syndrome: involvement of soluble mediators. Gut. 2009;58:196-201.

9. Di Gioacchino M, Masci S, Cavallucci E, et al. [Immunohistopathologic changes in the gastrointestinal mucosa in patients with nickel contact allergy.] G Ital Med Lav 1995;17:33. [Italian]

10. Minelli M, Schiavino D, Musca F, et al. Oral hyposensitization to nickel induces clinical improvement and a decrease in $\mathrm{TH} 1$ and $\mathrm{TH} 2$ cytokines in patients with systemic nickel allergy syndrome. Int J Immunopathol Pharmacol. 2010;23:193-201.

11. Drossman DA. The functional Gastrointestinal Disorders and the Rome III process. Gastroenterology 2006;130:1377-1390.

12. Calnan CD, Fregert S, Magnusson B. The international contact dermatitis research group. Cutis 1976;18:708-710.

13. Spiewak R. Patch Testing for contact allergy and allergic contact dermatitis. Open Allergy Journal 2008;1:42-51. 
14. Braga M, Quecchia C, Perotta C, et al. Systemic nickel allergy syndrome: nosologic framework and diet regimen. Int J Immunopathol Pharmacol 2013;26:707-716.

15. Bengtsson M, Ohlsson B, Ulander K. Development and psychometric testing of the Visual Analogue Scale for Irritable Bowel Syndrome (VASIBS). BMC Gastroenterol 2007;7:16.

16. Ware JE Jr, Sherbourne CD. The MOS 36-item short-form health survey (SF-36). I. Conceptual framework and item selection. Med Care 1992;30:473-483.

17. Dupy H. The psychological general well-being (PGWB) index. In: Wenger NK, Mattson ME, Furberg CD, Elinson J, eds. Assessment of quality of life in clinical trials of cardiovascular therapies. New York: Le Jacq Publishing Inc. 1984.

18. Butcher JN, Dahlstrom WG, Graham JR, Tellegen A, Kaemmer B. MMPI-2: manual for administration and scoring. Minneapolis: University of Minnesota Press 1989.

19. Balimane PV, Chong S, Morrison RA. Current methodologies used for the evaluation of the intestinal permeability and absorption. J Pharmacol Toxicol Methods 2000;44:301-312.

20. Rossi M, Sganga G, Mazzone M, et al. Cardiopulmonary bypass in man: role of the intestine in a self-limiting inflammatory response with demonstrable bacterial translocation. Ann Thorac Surg 2004;77:612618.

21. Perino A. Nickel and food. Int J Immunopathol Pharmacol 2005;18(4 suppl):15-17.

22. Simrèn M, Månsson A, Langkilde AM, et al. Food-related gastrointestinal symptoms in the irritable bowel syndrome. Digestion 2001;63:108115.

23. Lovell RM, Ford AC. Effect of gender on prevalence of irritable bowel syndrome in the community: systematic review and meta-analysis. Am J
Gastroenterol 2012;107:991-1000.

24. Hayes PA, Fraher MH, Quigley EM. Irritable bowel syndrome: the role of food in pathogenesis and management. Gastroenterol Hepatol (NY) 2014;10:164-174.

25. Iacovou M, Tan V, Muir JG, Gibson PR. The Low FODMAP diet and its application in east and southeast Asia. J Neurogastroenterol Motil 2015;21:459-470.

26. Staudacher HM, Whelan K, Irving PM, Lomer MC. Comparison of symptom response following advice for a diet low in fermentable carbohydrates (FODMAPs) versus standard dietary advice in patients with irritable bowel syndrome. J Hum Nutr Diet 2011;24:487-495.

27. Böhn L, Störsrud S, Liljebo T, et al. Diet low in FODMAPs reduces symptoms of irritable bowel syndrome as well as traditional dietary advice: a randomized controlled trial. Gastroenterology 2015;149:1399-1407.e2.

28. McKenzie YA, Alder A, Anderson W, et al. British Dietetic Association evidence-based guidelines for the dietary management of irritable bowel syndrome in adults. J Hum Nutr Diet 2012;25:260-274.

29. Cazzato IA, Vadrucci E, Cammarota G, Minelli M, Gasbarrini A. Lactose intolerance in systemic nickel allergy syndrome. Int J Immunopathol Pharmacol 2011;24:535-537.

30. Camilleri M, Madsen K, Spiller R, Greenwood-Van Meerveld B, Verne GN. Intestinal barrier function in health and gastrointestinal disease. Neurogastroenterol Motil 2012;24:503-512.

31. Lackner JM, Ma CX, Keefer L, et al. Type, rather than number, of mental and physical comorbidities increases the severity of symptoms in patients with irritable bowel syndrome. Clin Gastroenterol Hepatol 2013;11:1147-1157.

32. Folks DG. The interface of psychiatry and irritable bowel syndrome. Curr Psychiatry Rep 2004;6:210-215. 